\title{
ON PASIGRAPHY.
}

\section{ITS PRESENT STATE AND THE PASIGRAPHIC MOVEMENT \\ IN ITALY.}

$\prod \mathrm{HE}$ following communication was delivered in the German lanCongress of Mathematicians, first Section (for Arithmetic and Algebra). The Congress, which was attended by over 240 persons from nearly every civilised part of the globe, proved to be a remarkable success, though, owing to the fact that most of the British and American mathematicians were on their way to the Meeting of the British Association at Toronto, the English-speaking element was but scantily represented, there being only ten such persons present at the most. The next congress is to take place at Paris in 1900. The idea of starting such a congress having already been mooted at the meeting of the German Society of naturalists and physicians at Frankfurt a. M. in 1896, it ripened into a workable shape. There the opinion prevailed that the English language, being neutral ground between the French and the German, would be elected as the official means of communication, agreeably to which opinion the author had prepared his paper in English. ${ }^{1}$ We are glad to put the original-since but slightly altered-before our readers nearly at the same time that the Reports of the Congress appear.

1 The editors have been careful to preserve all the stylistic and typographical details of the original MS. of Professor Schröder. $-E d$. 
At an international Congress of Mathematicians there is in my opinion scarcely any topic more worthy of discussion, than that of Pasigraphy. For the aim of this novel branch of Science is nothing less than the ultimate establishment of a scientific Language, entirely free from national peculiarities, and through its very construction conveying the foundation of exact and true philosophy.

Such a language of course cannot be created at once for the whole realm of human thought. Its most important and hitherto mainly realised parts appear to be those which concern the fundamental notions of pure Mathematics, especially Logic, Arithmetic, Geometry.

I shall chiefly confine myself to some of these departments.

Time will not permit me to enter into an historical exposition. Suffice it to bring to recollection, that the pasigraphic discipline was clearly foreseen and postulated by Descartes, and that it formed an ideal hovering before the mind of LEIBNIz during his whole life. As my accomplished friend Signor Peano has recently pointed out, Leibniz so much cherished and appreciated the idea, that he says : except the founder of a religion or the ruler of a state - praeter Prophetam ac Principem-no person could better serve humanity than he who would realise that ideal-then so far away and actually not much more than a dim concept.

Leibniz also complained of the very small interest his contemporaries exhibited in the matter. The same complaint would in most quarters prove just as well founded now-a-days. However I venture to trust, that on the present occasion I may be fortunate enough to arouse some enthusiasm for this very important subject, which now appears to have entered upon a very promising stage.

Still at the outset it is necessary to contradict Signor Peano's statement of 1894 in his "Introduction au formulare de mathématique," p. 52, that: "Le problème proposé par Leibniz est (donc) résolu." With this sanguine dictum he-as we shall see-altogether anticipated the actual and impending achievement of pasigraphic science. For when his assertion was uttered, not even the indispensable means for attaining the goal had then been secured 
or rendered generally accessible, as they are at present. But even at this date there is yet much hard work to be gone through.

The problem to be solved for any given branch of science amounts to: expressing all the notions which it comprises, adequately and in the concisest possible way, through a minimum of primitive notions, say "categories," by means of purely logical operations of general applicability, thus remaining the same for every branch of science and being subject to the laws of ordinary Logic, but which latter will present themselves in the shape of a "calculus ratiocinator." For the categories and the operations of this "lingua characteristica" or "scriptura universalis" easy signs and simple symbols, such as letters, are to be employed, and-unlike the "words" of common language - they are to be used with absolute consistency (with perfect "Konsequenz," as we Germans say, or mathematical strictness, "Strenge").

It is almost superfluous to emphasise on how much higher a level this, our logical, aim stands, as compared with the merely linguistic endeavors of the Volapükists for instance, who are only striving to create means of mutual comprehension among the users of different languages, and the very mention of whom nearly amounts to a degradation of our object.

It may once for all be explicitly stated, that the pasigraphic language is not in the least destined ever to be spoken, but only to serve and forward on account of its logical structure the purposes of Science; first of all of that science, which the ancient Greek called "the science (katexochen)," Mathesis, and next: of Logic and an exact Philosophy, so long lacking, and hence to be hoped for-at last !

As an individual opinion of mine, perhaps not as yet shared by many, I may be permitted to state, by the way, that I consider pure Mathematics to be only one branch of general Logic, the branch originating from the creation of Number, to the economical virtues of which is due the enormous development that particular branch has been favored with in comparison with the other branches of Logic that until of late almost remained stationary. This view is confirmed by the fact, that under the pasigraphic aspect Arith- 
metic can do without any peculiar categories or primitive notionsthose of general logic sufficing to compose all its notions (such as multitude, number, finiteness, limes, function, Abbildung or oneto-one correspondence, addition, etc.).

If we limit our considerations to purest Mathematics it is indeed already becoming manifest-chiefly owing to the development which Charles S. PEIRCE's Logic of Relatives has experiencedthat all its notions, as well as those of Logic in general, are reducible to only five primitive notions or categories in the Aristotelian and Kantian sense.

Before these are presented a remark is needed.

The minimum number of indispensable symbols will exceed the said number 5 of the categories, some of which must find a representation by symbols doubly: just as in arithmetic neither of the two signs

$$
+ \text { and } \Sigma
$$

can in the long run or eventually be dispensed with, notwithstanding that both merely serve to represent the unique notion of an arithmetical sum.

Besides, these categories do not constitute the whole of the system of fundamental denotations. Whereas for instance parentheses or brackets form a very important and practically indispensable element of denotation, yet do not represent any notion at all and in themselves are devoid of meaning. (As is well known, brackets only serve in our symbolic language, as in Algebra, to characterise any compound of symbols, when included by them, as forming one name.) Moreover, we are constantly led to employ letters in the quality of general symbols, ready for such use, because of their having no fixed meaning attached to them.

This settled, the 5 categories or primitive notions of general logic with the inclusion of arithmetic are those which form the upper line in the following set:

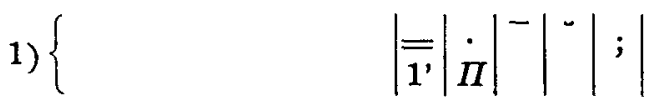

- the two first ones, as may be seen, being doubly represented, the 
second one even trebly, whereas the multiplication point (between letters) may as well be omitted, the result being a juxtaposition.

The first, being the well-known sign of equality, is in general logic to be interpreted in a much more restricted sense than in mathematics, viz., to mean identity or sameness; its equivalent 1' (a "one" with an apostrophe,-I pronounce it for shortness: oneap), puts forth the same category of identity as a relative term, destined to represent the class of things that are "equal to-" or "identical with", which sign sometimes also may be translated simply by the word "itself" = selbst $=$ le même $=$ lo mismo.

The second or multiplication point is used in general logicwholly independently of its arithmetical meaning-to express the category of intersection, Schnitt, since its office is always to denote that which is common to both the terms joined (and separated) by it. The $\Pi$ is then, analogically as in arithmetical analysis, employed for indicating "identical products" resulting from the operation of such intersection. $a \cdot b$ or $a b$ means: what is (at once) $a$ and $b$.

Our third category, to be represented by an overstroke, is the well-known logical operation of denial or negation. The sign-to speak more exactly-is intended to indicate its result, the negate. If $a$ means anything, then $\bar{a}$ denotes what is not- $a$. Evidently negation is a primitive notion or category, incapable of experiencing a formal definition. In lieu of the lacking definition, the so-called logical "principles" of contradiction and excluded middle step in to fill the gap. And by the bye be it said that similarly all principles of Logic as well as of Arithmetic would prove on examination to be mere substitutes for definitions (Peirce) and do not bear the character of axioms at all. (As is generally recognised, not every thing from the outset can be defined, since every definition has to rely on previous other notions, or categories already given.)

Our fourth category, represented by a crescent (to be placed over any letter), is that of conversion: if $a$ means cause of-, then $\breve{a}$ (a-converse) will denote effect of-, when $c$ denotes child of, then $\check{c}$ is to denote parent (i. e., father or mother) of-. I purpose to return to this point.

The fifth category, which I represent by a semicolon (Strich- 
punkt), is that of relation in general; the usual translation into words, of our semicolon being the particle "of," equalling "von," same as "de," the well-known predicate of nobility. If $a=$ amans means lover and $b$ means benefactor, then $a ; b$ denotes: lover of a benefactor. The operation consisting in the use of this sign is called relative multiplication or composition.

These five categories and their seven signs essentially suffice to embody all the fundamental notions of Logic and Arithmetic,as will be seen afterwards: I shall have to justify this apparently very daring assertion at least to some extent here in detail.

But if theoretically they prove sufficient, in practice it will not answer to restrain ourselves to their exclusive use. In order to avoid extreme cumbrousness, to secure the benefit of terseness or brevity and to facilitate clear surveys, also out of regard to symmetry, we are compelled immediately to supplement the foregoing system of juxtaposed signs.

The following three lines show how the 18 symbols of the succeeding set, which are forming our complete system of denotation (in general Pasigraphy), reduce to our five categories.

11 supplementary definitions:

2) $\left\{\begin{array}{c}0=a \cdot \bar{a}, 1=\overline{0}, 0^{\prime}=\overline{1^{\prime}}, a+b=\overline{\bar{a} \cdot \bar{b}}, \quad \Sigma a=\bar{\Pi} \bar{a}, a+b=\overline{\bar{a} ; \bar{b}}, \\ (a \neq b)=(a=a \cdot b),(a \notin b)=\overline{a \neq b},(a<b)=(a \neq b) \cdot(b \notin a), \\ (a \neq b)=\overline{a=b},(a \nless b)=\overline{a<b} .\end{array}\right.$

The 18 signs:

3) $0,1,+, \cdot, \Sigma, \Pi, 0^{\prime}, 1^{\prime},-,-,\left(^{*}\right), f, ;, \neq,=,<, \neq, \neq, \measuredangle$.

Let us deal with these rapidly.

By the first of these equations is defined the logical notion of Nothing, which in general Logic is to be denoted by the cipher naught, 0 . Whenever the need should arise to use the same sign for the number naught or zero, very much to be distinguished therefrom, I prevent their being confounded by putting a dot over the latter: $\dot{0}$. "Nothing" is here defined as that which is at once $a$ and not- $a$, no matter what $a$ may mean.

The next equation defines "something" as not-nothing. This notion comprises everything of which it is possible to speak, the Thinkable, and the sign 1 (one) thus is to represent in general 
Logic the Totum, the notion of $A l l$, the Whole, der Denkbereich, say the "Universe of discourse." This also may occasionally be further restricted for the purpose of any special investigation. For preventing its sign from being confounded with the number one (1) as only can, and seldom will, occur in researches of a mixed character, both logical and arithmetical, it is my practice in the latter case to put a dot over it. (Similarly in such a case I employ the sign $\times$ for indicating arithmetical multiplication and a larger + for the arithmetical addition.)

The third equation 2) defines the relative term "different from " or "other than-" as being not identical with-, and introduces for designating it an apostrophised naught, to be spoken naught-ap. If this relation is to be stated between two terms, it is already customary (in German mathematical periodicals) to express it by a sign of equation cancelled by a down stroke and thus negatived in effigy, + thus meaning unequal-see the definition last but one.

The fourth and fifth equation define the "identical sum" or logical aggregate (Inbegriff, Gesamtheit) to be denoted in general Logic by the signs borrowed from Arithmetic + and $\Sigma$. $a+b$ is here to express that which is not at once not- $a$ and not- $b$; id est : what is either $a$ or $b$, perhaps both.

The sixth equation 2) introduces a sign $f$ "plus with a scorpion tail to the left" that I pronounce with the Italian word for + , viz., as più, for designating a relation very strange to ordinary thinking: a relation hitherto without a name. $a+b$ is to represent that which is not a not- $a$ of a not- $b$, and this amounts to: an $a$ at any rate of everything but $b$ 's (no matter, whether it is an $a$ of $b$ 's also, or not). The operation of connecting $a$ with $b$ by means of this sign $f$, which thus results in the formation of the notion $a_{f} b$, is called relative addition. The introduction of this apparently somewhat intricate unfamiliar notion is dictated by a regard for symmetry. In Logic whenever a class $a$ is formed, the same interest as to the individuals within is due to those without this class, i. e., to the not-a. There is a duality of notion (dualism) between "containing" and "being contained in," $\neq$ and $\neq$ - see further on. Thus the frelation corresponds to the category of (;) exactly 
in the same way as product and sum or the . and + , as the particles and and or correspond with each other, surely none of which could be missed. To give an example at once, if $t$ means Teiler, divisor of-, and if we restrain the Universe of discourse to the common numbers, then $t_{f} 0$ will express : what is a divisor of every number, save nothing or no number, and this simply means: a divisor of every number. Such indeed is the numerical unit, the number one, and none other.

Our next definition introduces the all-important notion of $\mathrm{im}$ plication or inclusion, the being contained in- as a part anyhow (that is may-be as a proper or genuine part, echter Teil, or may even be as the whole itself). The implication or subsumption $a \leqslant b$, to be read as " $a$ is contained within $b$," or " $a$ is part of $b$," appears to be explained here by : $a$ is identical with that, which is at once $a$ and $b$. My implication sign $€$ (in German to be read "eingeordnet") generally translates the copula "is," "est" of a categoric statement, and also, when placed between statements $a$ and $b$, presents itself as the sign of inference or illation: for though the conclusion is in a certain sense implied by or involved in the premises, however conversely, if $b$ follows from $a$, the class of occasions when $a$ holds good, will be contained within the class of cases where $b$ holds. The subsumption $a \neq b$ then may be read as : whenever $a$ is true then $b$ is true.

The next definition only introduces the denial of the foregoing relation : being not contained in-, (I need not enlarge thereon) in the same way as the last definition introduces the denial of the one we only have yet to discuss.

In the remaining definition 2), also an important one, is explained the relation of being contained in- as a proper part : $a$ is contained in $b$ as such, $a<b$, whenever $a$ is contained in $b$, whilst $b$ is not contained in $a$ (or is other than $b$ ).

This again settled so far, we are in possession of and we com. mand the complete denotation-system of general Logic, which consists of these eighteen signs 3 ), henceforth rendered legitimate for the use of Pasigraphy by their reduction to the five categories.

The system of denotation expounded is that which has natur- 
ally arisen from the profound and persistent investigations, extending through nearly half a century, of men of the genius of DE MORGAN, BOOLE and most of all of one of the keenest American thinkers : Mr. Charles S. Peirce. ${ }^{1}$ In working over his theory at large I have but slightly and never without intrinsic reasons, modified his (or Boole's) denotations, deviating only slightly, at least as compared with the divergencies shown by every system of denotation that derives from other sources, especially that of Signor Peano and the Italian school. I shall call the former for simplicity's sake "Peirce's system" (omitting the addition "as modified by me"). For the benefit of those who are already familiar with the symbolism of the latter (Peano) and his most numerous, active and skilful adherents it may at once be stated that our signs
4) $\{$ corrt
$0, \quad 1,+, ., \Sigma, \Pi, \quad \bar{a}, \quad €$

By the way, since the signs $\Sigma$ (and $\Pi$ ), as is well known, have to serve as the bearers, scaffolding, support, frame for the shifting suffix (Summationsvariable), which is to pass through a series of values, and besides for the limits (upper and under) of that sum or series, the substitutes above given by Peano for these $\Sigma$ and $I I$ appear to be chosen still less happily, than if in arithmetical analysis we should propose to replace the $\Sigma, I$, generally in use, by a f' and $x$ '. Such an "emendation " turning out to be but a deterioration, would in German be ironically styled: "eine Verschlimmbesserung."

Touching the essential divergence, that Peano's denotationsystem lacks our fifth category "of," the most important of all, and that in consequence it cannot show any signs corresponding to our relative operations ( $f$ and ;), I shall have a few words to say further on.

Now the calculus ratiocinator ruling, nay governing, our categories and fundamental operations, to the laws of which these primitive elements of thought are of necessity subject, is none other than Peirce's "Algebra of Relatives," a discipline (branch of science)

\footnotetext{
${ }^{1}$ Not, bowever, infallible, as will be seen on a future occasion.
} 
crowning the edifice of the "Algebra of Logic" and comprising as well the statement-calculus as the class-calculus-both as very subordinate parts.

Almost everything may be viewed as, or considered under the aspect of, a (dual or) binary relative, and can be represented as such. Even statements submit to be looked at and treated as binary relatives. Classes, assemblages (Mengen, ensembles) or absolute terms may be thus presented.

And since in ordinary as well as in scientific thinking the relative notions by far prevail over the absolute ones, which latter, over and above, are eventually comprised in and superseded by them, it is evident, that the Logic of the relative notions, Relatives, must form the indispensable base and underlie every successful attempt at Pasigraphy.

In the fact that traditional Logic so long confined itself to the absolute notions with the meagre categories of "all," "some," and "none" is to be perceived an essential cause for its stagnation, that undeniable standstill, which yet entitled KaNT in his time to make the assertion: that during the two thousand years since ArisTOTLE Logic had not accomplished any real progress. This would now no longer accord with the facts.

And as for the present time, it may warningly be said that whosoever, while aiming at our logical ends, tries to erect the building on a narrower ground than that created and offered by the De Morgan-Peirce theory (which reposes on the general notions of relation, Relative and composition), such as for instance would be furnished by the introduction and admission among the categories merely of the notion of "function," or say of "transformation," or else of "(one to one) correspondence"- these altogether being by far more special, and only particular cases of that general notion of Relatives-whoever contents himself with logograms for any of such special notions will preclude himself from participating in and benefiting from the above already highly developed theory; he will bar, nay block, for himself the way to expeditious progress.

Let us now illustrate the scope and purport of our novel Logic of Relatives, and therewith demonstrate, at least to a large extent, 
the sufficiency, here claimed, of the set of our five primitive notions 1) for building up the complete body of the fundamental notions of Arithmetic. I shall therefore put before the reader, arranged in several groups, the pasigraphic representation and definition of not a few of its most essential notions-to be aided by subsequent remarks.

5) $\left\{\begin{array}{l}\text { biet, System, Menge, ensemble, insieme })=(a ; 1=a)= \\ =0+\check{a}+a+0 .\end{array}\right.$

( $a$ is a class, assemblage, collection, set, an absolute term, Ge-

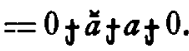

6) (num. $a=\dot{0})=(a=0)=0, \bar{a}+0$.

7) $\{$ (num. $a=1)=(a$ is an individual, element, constant function, 7) $\left\{\begin{array}{l}\text { may be a single number })=\left(0^{\prime} ; a ; 1=\bar{a}\right)= \\ =\left(0^{\prime} ; a=\bar{a}\right)=\left(a-0^{\prime} ; a\right)=\check{a} ;\left(1^{\prime} \jmath \bar{a}\right) .\end{array}\right.$

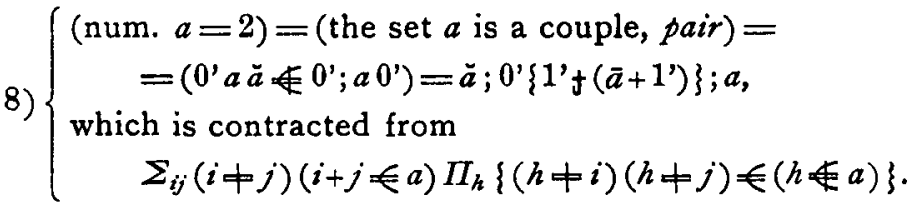

9) (num. $a>3)=\left(0^{\prime} a \check{a} \cdot 0^{\prime} ; a 0^{\prime}+0\right)=\check{a} ; 0^{\prime}\left(0^{\prime} ; a 0^{\prime}\right) ; a$.

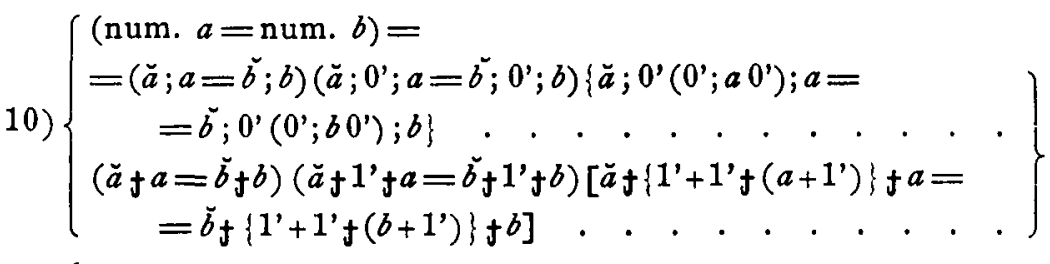

11) $\left\{\begin{array}{c}(a \text { is gleichmächtig, of equal might with, } b \text {, according to Herrn } \\ \text { G. Cantor's terminology : } a \text { is "equivalent" } b)=\end{array}\right.$ $=(a \sim b)=\sum_{s}\left(z ; \check{z}+\check{z} ; z \neq 1^{\prime}\right)(b=z ; a)(a=\check{z} ; b)$

12) $\left\{\begin{aligned} a & \text { is } \bar{\infty})=(\text { the collection } a \text { is finite })= \\ & =\Pi\left\{\left(z ; \check{z}+\check{z} ; z \neq 1^{\prime}\right)(a \neq \check{z} ; a) \neq(a \neq z ; a)\right\} .\end{aligned}\right.$

13) $\left\{\begin{array}{c}(a \text { is } \infty)=(\text { the set } a \text { is "actually infinite," transfinite })= \\ =\sum\left(z ; \check{z}+\check{z} ; z \neq 1^{\prime}\right)(z ; a<a \neq \check{z} ; z ; a) .\end{array}\right.$

14) $(f$ is a function $)=(f ; \tilde{f} \neq 1, \neq \tilde{f} ; f)$.

15) ( $s$ is a substitution, permutation) $=\left(s ; \breve{s}=1^{\prime}=\breve{s} ; s\right)$.

16) $\left\{\begin{array}{l}(i \text { is an } a \text { of } j)=(i \neq a ; j)=a_{i j}=\check{i ;} a ; j \\ \quad-i, j \text { representing individuals, compare } 7) .\end{array}\right.$ 
17) $\left\{\begin{array}{c}\text { (The set } a \text { is put in simple order according to the principle } x)= \\ =(x ; x \leqslant x=0 \text { ' } a \cdot a \ddot{x}) .\end{array}\right.$ 18) $\left\{\begin{array}{c}\text { (The whole universe of thought is marshalled in a simple or- } \\ \text { der, in a file or succession by } x)=\left(x ; x \neq x=0^{\prime} x \tilde{x}\right) .\end{array}\right.$

Dwelling for a moment upon the above, we shall here perceive represented and defined through the medium of the fund or capital ${ }^{1}$ of denotation hitherto secured, quite a series of notions, fundamental for Arithmetic and Mathematics in general.

Before considering some of these in detail, a few remarks are required.

From 5) until 9) and at 16) I have, as concluding term, given the definition itself also in the shape of a binary relative. These then are "prominent (ausgezeichnete)" relatives, being only capable of one or other of the two "truth-values" (or "absolute moduli") 0 and 1.

The "class, assemblage, system, collection or set" having been defined by 5 ), I did not, from the middle line of 7 ) onwards, explicitly state that $a$, and may be $b$, ought to represent assemblages or classes, leaving this to be tacitly understood for fear of overloading the formulæ.

We next hit upon the definition of the lowest natural (i. e., positive integral) numbers $\dot{0}, \dot{i}$, and 2 . Verbal Logic has hitherto proved incapable of defining even the casus singularis.

It is, of course, not practicable to enter upon the explanation and establishment of all these definitions one by one. I should like, however, as an example which can be easily understood, to point out the genesis of the definition of the number 2 . The last line of 8) literally shapes into expression: There is an element $i$ and again an element $j$ ("another element"), differing from the former, such that (both are) each of them is contained within the set or assemblage $a$, whilst every element $h$ differing from the one and the other $i, j$ will not be contained within $a$. Evidently this is indispensable and sufficient whenever the set $a$ shall consist of ex-

\footnotetext{
1"Capital" is here to be taken in the sense of Adam Smitb and Political Economy.
} 
actly two elements. But according to the rules or Laws of the Algebra of Relatives, as developed in my book, the preceding prolonged intricate statement easily condenses into the forms given above it. Eventually there is but an expenditure of say six letters to be spent on defining "a pair" or the casus dualis. I wonder whether that can be styled waste!

In 11) besides the (relative) notions of " equal might" (between sets) you may observe as being pasigraphically defined the notion of "Abbildung" or one-to-one correspondence, the latter standing behind the $\Sigma$. That is to say : the sets $a$ and $b$ are to be called of equal might (multitude), whenever there exists a relative $z$ which in that sense images (projects) the one set on the other.

12) gives the definition of finiteness (of a set). This, in accordance with Peirce, may be given independently by expressing the fact, that in passing from one to another through the elements of the set one must necessarily come back to an element already passed.

13) gives the definition of infinity, likewise independently in the usual manner: as the quality of the set to be capable of being imaged (projected) on a proper part of itself.

Both notions can be shown (by mere calculation) to be but negations of each other, their definitions being contraposed to one auother. ${ }^{1}$ Neither of the two definitions exhibiting or containing the least particle of negation, they furnish a good example for illustrating the falsity of the doctrine, still current among professional philosophers, of a distinction being logically possible between notions or marks (Merkmale, notae) positive and negative in themselves. I challenge any one of them, including Mrs. Franklin-Ladd (compare her review of my Vol. 1 in Mind), to decide which of the notions "finite" and "infinite" is the positive one and which the negative, whilst for such decision supplying reasons that appertain to the domain of Logic.

10) gives the explicit condition for equalness of number, i. e., for the fact that two sets $a$ and $b$ contain the same number of ele-

${ }^{1}$ See my papers in the Nova acta Acad. Leop. Carol., Vol. 71, 1898. 
ments, or that within each equally many individuals may be counted. This, of course, presupposes the finiteness of both sets.

The condition is set out as an infinite series of partial conditions, and in the shape of a relation between both sets. It may well be seen therefrom how well-founded is Herrn Dederind's remark: that the notion of the "number" of things is wrongly believed to be a simple one.

Into 17) the notion of the "simple order" has pasigraphically condensed itself from the marks that the Signori ValLati and Burali-ForTi have pointed out one by one, endeavoring to invest them with and dress them in the symbolism of the Italian schoola symbolism apparently not equal to such tasks and no match for our pasigraphic symbolism, supported as that is by so powerful a discipline as Peirce's Algebra of Relatives. It reminds one of stenographic briefness to notice that for a full investment and adequate expression the statement 18) an expenditure of only five letters is needed. Nevertheless, every person versed in relative Logic can read therefrom all the qualities of a simply ordered Whole, either whilst skilfully deducing them by conclusions to be drawn of necessity, or even at first sight, by mere inspection. Of course, supposing a flourish of any kind to be made, such merely shorthand logogram ("Schlüssel") would easily beat in briefness our pasigraphic expression, but then that which is most valuable in the latter, i. e., the fact (last mentioned) of its containing visibly condensed within itself all the marks of the notion to be represented by it, and therefore of its being capable to yield them again at any moment, would be forfeited.

With respect to the notion of "order " and its different " types" it would be well worth while to enter and enlarge upon the pasigraphic representation of the many notions with which Herr G. CANTOR has here enriched Science. We might, for instance, next show that the postulation: "there exists within the set $a$, ordered by the principle $x$, an element of lowest range" presents itself thus $a \notin 0$ ' $\bar{x} ; a$, and that $a\left(\breve{x}_{j} 0\right)$ is the expression of this "initial" element, and so on. But the time at our disposal will not allow me to continue in that direction. 
Similarly as in the foregoing we could now also pasigraphically define the statement

(num. $a=$ num. $b+\dot{1}$ ).

One would thus for the realm of numbers succeed in constituting a certain Relative :

$$
g,=\text { by } i \cdot g r e a t e r \text { than- }
$$

by means of which, though not very simply, is to be represented also the Relative :

$$
t=\text { Teiler von-, divisor of-, }
$$

or else, if it be preferred, this one : $\bar{t}=$ multiple of-, Vielfaches von-.

Then we shall have :

$$
\dot{i}=t_{f} 0, \dot{0}=\check{t}_{f} 0
$$

and again, for example:

$$
\begin{aligned}
& r=\text { (relatively prime with-, teilerfremd mit- })=\ddot{t}_{\mathrm{f}}(\dot{i}+\vec{t}) \text {, } \\
& (m \text { is prime with } n)=(m \neq r ; n)=\check{m}\left\{\check{t}_{f}(\dot{1}+\bar{t})\right\} ; n \text {, } \\
& \text { Prime number }=\left(1^{\prime}+\check{t}\right)+\dot{1}=(t+r) \dagger 0 \text {, } \\
& \text { (Greatest common divisor of } m \text { and } n \text { ) }= \\
& =t ; m \cdot t ; n \cdot\{\ddot{t}+\bar{t} ;(m+n)\} \text {, }
\end{aligned}
$$

And thus to be continued at pleasure. With these and suchlike forms it is possible to calculate, and inferences regarding the notions they represent, may be drawn and extracted from them. This latter could not be effected with mere shorthand logograms, such as is for instance Peano's $D(m, n)$ for the notion next to the last in 23).

The notion of absolute prime number being doubly represented above (for the realm of the whole numbers), the first representation states: prime number is a number which stands to each num. ber, except the $i$, in the relation of either being identical with it or being no multiple of it. The second states: prime number is what to every number (without exception) stands in the relation of either being a divisor of it or being relatively prime with it. And, on the strength of the pasigraphic structure (not here given) of the Relative $t$ itself, either one of these two representations will be capable, moreover, of being transformed into the other. 
For the sake of throwing a momentary glance on topics other than Arithmetic in this place, supposing the universe of discourse 1 to mean Space, the definition of a geometrical point may be given : 24) $\left\{\begin{array}{l}(z \text { is a point })=(z \neq 0) \prod_{u}\{(z \neq u)+(z \neq \bar{u})\} \\ \text { or, in another form (after Peirce) }=(z \neq 0) \prod_{u}\{(u<z) \in(u=0)\} .\end{array}\right.$

In its first shape our definition settles the "point" to be such a part of space, differing from nothing, which to any part $u$ of space stands in the relation either of being wholly contained within it, or being wholly without it, that is to say, being wholly contained within the remainder of space $\bar{u}$. I leave to the reader the interpretation of the second form of definition, which has been already reduced by me to the former in my Vol. 2 .

Finally a word anent the Pasigraphy of human relationships embracing as well those of consanguinity as those of affinity and forming no unimportant chapter in the corpus juris for the student of law. In addition to a few of the signs of General Logic aboveset out, there are only requisite two specific symbols of Relatives, for representing discriminately and exhaustively all these relationships in the concisest possible shape. These two are:

and

$$
m=\text { male (an absolute term), }
$$

$$
c=\text { child of- (a relative one). }
$$

Mankind consisting of two sexes then $\bar{m}=$ not-male will denotefemale, and $\breve{c}$, as before mentioned, will equal "parent of-." The universe of discourse $1=m+\bar{m}$ then consists of the Persons of human society in the Past, Present, and Future. However, for rendering fully accessible to our pasigraphic system also the relations of affinity (i. e., those by marriage only), to every childless married couple must be ascribed one "potential child." True, that for completely realising the ideal of Pasigraphy it might be demanded that, again, the notions "male" and "child of-" themselves should be reduced to primitive notions of a simpler breed. But such a thing might only be hoped for when Zoölogy and Physiology should have developed to a much higher degree of perfection. Meanwhile something yet is to be won if we plainly admit these two notions $m$ and $c$ as primitive notions and henceforth use them as building stones. 
Then the following will be the pasigraphic representation of sundry relations :

( Maybe only half-) brother or sister $=0, . c ; \dot{c}$,

Full brother or sister (Geschwister) $=0, . c ; m \check{c} . c ; \bar{m} \check{c}$,

Fullbrother $=0^{\prime} m . c ; m \check{c} . c ; \bar{m} \check{c}$, Full sister $=0^{\prime} \bar{m} . c ; m \check{c} . c ; \bar{m} \check{c}$,

Stepchild $=\tilde{c} \cdot c ; \breve{c} ; c, \quad$ Father $=m \breve{c}, \quad$ Mother $=\bar{m} \breve{c}$,

Consort $=0^{\prime} \cdot \check{c} ; c, \quad$ Husband $=0^{\prime} \cdot m \check{c} ; c, \quad$ Wife $=0^{\prime} \cdot \bar{m} \check{c} ; c$,

Nephew or niece $=c ; 0^{\prime}(c ; \breve{c})$, Mother-in-law $=\bar{m} \breve{c} ; 0^{\prime}(\breve{c} ; c)$.

All these multifold connexions have been most profoundly studied by Mr. Alexander Macfarlane who has, for instance, answered the question: which relationships (being of the second degree) are excluded (prohibited from existing) by the English Law that forbids a man to marry his deceased wife's sister. With suchlike expressions as those already given any kind of problems may also be solved mechanically, by mere calculation, as, for example, this : a lady, questioned about a photograph in her album, replies : "you know that I have no daughters. Well, this person's daughter's son is the father of one of my grandchildren." How was the original of the portrait related to the lady?

Macfarlane, however, because of his repudiating Peirce's Algebra of Relatives, or at least abstaining from its use, did not clear a certain reef. Whereas in the expressions by him established, that yet are somewhat different from the above, he did not succeed in excluding their "reduced meaning"-as he chose to call it.

The gist of the situation may already be clearly perceived in the well-known riddle for children: My father has a son who still isn't my brother; who is it?

The "reduced meaning" of child of the parents of somebody is this somebody him- (or her-) "self" ( 1 '), and therefore the supplementary appending of the $\operatorname{sign} 0^{\prime}=$ " another than." to $c ; \check{c}$ is indispensable for correctly forming the notion of brother or sister.

Turning from these special investigations of English origin and leaving untouched several isolated attempts (as for instance that of Herr FREGE, who heedless of anything accomplished in the same direction by others, took immense pains to perform what had already been much better done and was therefore superseded from 
the outset, thus delivering a still-born child) save the fundamental work of Mr. PeIrce in the United States and his German and English precursors among whom BOOLE and DE MORGAN deserve first mention, the aims of Pasigraphy hitherto have found assiduous promoters only in Italy.

The meritorious periodical Rivista di Matematica, edited for five years by the eminent mathematician Signor Peano, our chairman on this occasion, together with the supplementing Formulario, are mainly devoted to its purposes. And in this and other periodicals through a group of keen Italian investigators quite a series of branches of Analysis and Geometry has been worked over with pasigraphic intention and enormous application. All that can be done with the Boole-McColl "calculus of equivalent statements," and that is certainly much, appears almost wholly to have been thereby accomplished-though, regrettably, in a greatly diverging system of denotation. On the other hand, in its general features the present phase of the Italian pasigraphic movement is characterised by the non-use hitherto of Peirce's Algebra of Relatives. Against turning the latter to profitable account the denotation system adopted by the Italian school indeed seems almost to form an obstacle. Their capital of denotation lacks the most general primitive notions, which in the Algebra of Relatives already exist and are tolerably well investigated with respect to the rules of their combination. For these missing categories numerously invented and arbitrary logograms (occupying 5 printed pages of Peano's Table des signes and still on the increase) prove insufficient substitutes and are but poor makeshifts. To conclude here, in short, I may venture to apply to them the parable, put forth by Professor Minkowski in his address when introducing the proceedings of our Section, concerning those who persist in still using sailing ships whilst steamboats have already been invented, constructed and are waiting at their service.

If I have successfully shown how with the same means the notion of infinity and of the greatest common divisor, equally well as that of mother-in-law, can be expressed, then surely it will be admitted that Pasigraphy has now indeed emerged from the status 
nascendi and that its ideal must have been realised at least to some extent.

In the cases-ever rare-when humanity has succeeded in essentially realising an ideal, as a rule its subsequent aspect will widely differ from the form in which it hovered before those who conceived it first. So in this case. Already we can say thus much, that Leibniz's prediction: "scriptura haec universalis aeque erit facilis quam communis" is scarcely likely ever to be fulfilled, and that Descartes's hope, that by its aid a peasant would then gain a deeper insight into things than is now possessed by a philosopher, will probably never be realised.

It is in the calculus ratiocinator that the difficulty lies! The higher parts of Logic present such an abundance of problems ranging among those of the very highest degree of intricacy, and mastering the Algebra of Relatives-accessible only to serious workers-is so little easy to attain that it may well never become common property, always remaining the privilege of but a few favored thinkers.

In conclusion, and returning once more to the 5 primitive notions 1), I have permitted myself, in selecting them, to be led by regards of convenience for the purposes of my lecture. I have been very far from implying, however, that their number may not possibly be further reduced. As a matter of fact our "category" - of conversion seems-by means of the definition

$$
(i \neq a ; j)=(j \neq \breve{a} ; i)
$$

wherein $i$ and $j$ in the sense of 7 ) represent individuals-itself to be reducible to the four remaining primitive notions, provided only that the whole set of the "Definitions" be systematically" arranged in a proper manner.

Then the "four elements"

$$
\text { same, and, not, of }
$$

or identitas, intersectio, negatio, relatio, in intimate association will both form life and sustain the world intellectual.

\section{ERNST SCHRÖDER.}

KARLSRUHE IN BADEN. 